Pacific

Journal of

Mathematics

NILMANIFOLDS AND ASSOCIATED LIE ALGEBRAS OVER THE INTEGERS

Bohumil Cenkl and Richard Porter

Volume 193 No. 1

March 2000 


\title{
NILMANIFOLDS AND ASSOCIATED LIE ALGEBRAS OVER THE INTEGERS
}

\author{
Bohumil Cenkl and Richard Porter
}

We consider the correspondence between nilmanifolds and Lie algebras with rational basis, and we define spectral sequences converging to the respective cohomologies. The $E_{2}$ terms of the spectral sequences are the cohomolgies of isomorphic graded Lie algebras.

Each nilmanifold gives rise to a Lie algebra with rational basis. We give an example which illustrates that not all such Lie algebras correspond to nilmanifolds. Given a Lie algebra with rational basis we give a construction that produces a nilmanifold with Lie algebra that is rationally equivalent to the starting Lie algebra.

\section{Introduction.}

In Section 1 we consider the correspondence between groups and Lie algebras over the reals and rationals. A nilmanifold is completely determined by its fundamental group - a finitely-generated, torsion-free, nilpotent group. In Section 2 we show how a nilmanifold determines a nilpotent Lie algebra with a finite rational basis; we give an example to illustrate that not every such Lie algebra comes from a nilmanifold. In Section 3 we define a spectral sequence for a nilmanifold. We prove that spectral sequence converges to the integer cohomology of the nilmanifold and has $E_{2}$ term isomorphic to the cohomolgy of a graded Lie algebra. In Section 4 we define the corresponding spectral sequence for the Lie algebra associated to a nilmanifold. The spectral sequence converges to the cohomology of the Lie algebra; the $E_{2}$ term is the cohomology of a graded Lie algebra, and if $\mathbb{A}$ is the smallest subring of the rationals containing the structure constants of the Lie algebra then with coefficients equal to $\mathbb{A}$ the $E_{2}$ terms of the spectral sequences of the nilmanifold and the Lie algebra are isomorphic. In Section 5 we give a construction of a nilmanifold from a nilpotent Lie algebra with finite rational basis; the Lie algebra associated to this nilmanifold is rationally equivalent to the original Lie algebra. 


\section{Nilmanifolds and corresponding Lie algebras over the reals and the rationals.}

A nilmanifold is the quotient of a simply-connected Lie group by a uniform discrete subgroup. A uniform discrete subgroup can be identified with a finitely-generated, torsion-free, nilpotent group. We denote such a group by $G$. Let $\mathcal{G}_{\mathbb{R}}$ be a $k$-dimensional, connected, simply-connected, nilpotent Lie group. Then $\mathcal{G}_{\mathbb{R}}$ can be identified with its real Lie algebra $\mathcal{L}_{\mathbb{R}}$ with bracket $[$,$] . With respect to any basis \left\{d_{1}, \ldots, d_{k}\right\}$ for $\mathcal{L}_{\mathbb{R}}$, the group $\mathcal{G}_{\mathbb{R}}$ can be identified with the set of elements $\xi_{1} d_{1}+\cdots+\xi_{k} d_{k}, \xi_{j} \in \mathbb{R}$, in $\mathbb{R}^{k}$. The Lie algebra $\mathcal{L}_{\mathbb{R}}$ has real structure constants and the group structure $*$ on $\mathcal{G}_{\mathbb{R}}$ (as well as on any subgroup) is given by the Campbell-Hausdorff formula:

Using the notation $a d(x)(y)=[x, y], a d^{2}(x)(y)=\operatorname{ad}(x)([x, y])=$ $[x[x, y]], \ldots$ we can write

$$
\begin{aligned}
& x * y=z(x, y), \\
& z(x, y)=\sum_{n=1}^{\infty} z_{n}(x, y) \\
& z_{n}(x, y)=\frac{1}{n} \sum_{p+q=n}\left(z_{p, q}^{\prime}+z_{p, q}^{\prime \prime}\right), \quad \text { where } \\
& z_{p, q}^{\prime}=\sum_{\substack{p_{1}+\cdots+p_{m}=p \\
p_{1}+\cdots+q_{m-1}=q-1 \\
p_{i}+q_{i} \geq 1, p_{m} \geq 1}} \frac{(-1)^{m+1}}{m} \frac{a d^{p_{1}}(x) a d^{q_{1}}(y) \cdots a d^{p_{m}}(x)(y)}{p_{1} ! q_{1} ! \cdots p_{m} !}, \\
& z_{p, q}^{\prime \prime}=\sum_{\substack{p_{1}+\cdots+p_{m}=p \\
q_{1}+\cdots+q_{m-1}=q-1 \\
p_{i}+q_{i} \geq 1, p_{m} \geq 1}} \frac{(-1)^{m+1}}{m} \frac{a d^{p_{1}}(x) a d^{q_{1}}(y) \cdots a d^{p_{m-1}}(y)(x)}{p_{1} ! q_{1} ! \cdots q_{m-1} !} .
\end{aligned}
$$

It is easy to find the first three homogeneous components of $z$. Namely,

$$
\begin{aligned}
& z_{1}(x, y)=x+y \\
& z_{2}(x, y)=\frac{1}{2}[x, y] \\
& z_{3}(x, y)=\frac{1}{12}[x,[x, y]]+\frac{1}{12}[y,[y, x]] .
\end{aligned}
$$

It is clear that $z(x, 0)=x, z(0, y)=y$, and $z(z(w, x), y)=z(w, z(x, y))$.

Malcev [3] showed that a group $\mathcal{G}_{\mathbb{R}}$ contains a uniform discrete subgroup $G$ if and only if there is a basis for $\mathcal{L}_{\mathbb{R}}$ with rational structure constants. Let $\left\{d_{1}, \ldots, d_{k}\right\}$ be such a rational basis. Then the set of elements

$$
\xi_{1} d_{1}+\cdots+\xi_{k} d_{k}, \quad \xi_{j} \in \mathbb{Q}
$$


has the structure of a Lie algebra $\mathcal{L}=\mathcal{L}_{\mathbb{Q}} \cdot \mathcal{L}$ as a subset of $\mathcal{G}_{\mathbb{R}}$ has group structure given by the Campbell-Hausdorff formula. We denote this group by $\mathcal{G}_{\mathbb{Q}}$. Since $\mathcal{L}$ is a nilpotent Lie algebra, a rational basis for $\mathcal{L}$ can be chosen so that $\left\{d_{i}, \ldots, d_{k}\right\}$ is a basis for an ideal $\mathcal{L}^{i}$ of $\mathcal{L}$, with $\left[\mathcal{L}^{i}, \mathcal{L}\right] \subseteq \mathcal{L}^{i+1}$ and $\mathcal{L}^{k+1}=0$. Such a basis is called a canonical basis for $\mathcal{L}$. It follows from the Jacobi identity that

$$
\left[\mathcal{L}^{i}, \mathcal{L}^{j}\right] \subseteq \mathcal{L}^{i+j}
$$

for $i, j \geq 1$. In particular, the bracket on $\mathcal{L}$ induces a graded Lie algebra structure on $\bigoplus_{i \geq 1} \mathcal{L}^{i} / \mathcal{L}^{i+1}$.

Let $\mathcal{G}$ be the group on the set of elements

$$
\left(x_{1} d_{1}\right) * \cdots *\left(x_{k} d_{k}\right), \quad x_{j} \in \mathbb{Q},
$$

with group structure given by the map $*$. The elements

$$
\left(x_{i} d_{i}\right) * \cdots *\left(x_{k} d_{k}\right)
$$

generate a normal subgroup $\mathcal{G}^{i}$ of the group $\mathcal{G}$.

$$
\mathcal{G}=\mathcal{G}^{1}>\mathcal{G}^{2}>\cdots>\mathcal{G}^{k}>1
$$

is a central series. The commutator $(a, b)=a^{-1} b^{-1} a b$ for any $a \in \mathcal{G}$ and $b \in \mathcal{G}^{i}$ belongs to $\mathcal{G}^{i+1}$, and the quotient groups $\mathcal{G}^{i} / \mathcal{G}^{i+1}$ are isomorphic to $\mathbb{Q}$.

Proposition $1([3])$. The groups $\mathcal{G}$ and $\mathcal{G}_{\mathbb{Q}}$ are isomorphic. The isomorphism is given by the maps

$$
\mathcal{G} \underset{\psi}{\stackrel{\varphi}{\rightleftarrows}} \mathcal{G}_{\mathbb{Q}}, \quad\left(x_{1} d_{1}\right) * \cdots *\left(x_{k} d_{k}\right) \underset{\psi}{\stackrel{\varphi}{\rightleftarrows}} \xi_{1} d_{1}+\cdots+\xi_{k} d_{k},
$$

where

$$
\xi_{j}=x_{j}+\varphi_{j}\left(x_{1}, \ldots, x_{j-1}\right) \quad \text { and } \quad x_{j}=\xi_{j}+\psi_{j}\left(\xi_{1}, \ldots, \xi_{j-1}\right)
$$

are rational polynomials.

Proof. See [3].

The explicit formulas for $\varphi$ and $\psi$ follow from the Campbell-Hausdorff formula.

Note that the maps $\varphi$ and $\psi$ give one-to-one maps of sets

$$
\mathcal{G}^{i} \underset{\psi}{\stackrel{\varphi}{\rightleftarrows}} \mathcal{L}^{i}, \quad i=1,2, \ldots, k .
$$

The group $\mathcal{G}$, as a set, can be identified with $\mathbb{Q}^{k}$ via the map $e: \mathcal{G} \rightarrow \mathbb{Q}^{k}$ given by

$$
e:\left(x_{1} d_{1}\right) * \cdots *\left(x_{k} d_{k}\right) \longrightarrow x=\left(x_{1}, \ldots, x_{k}\right) .
$$

$e$ induces on $\mathbb{Q}^{k}$ a group structure by the operation

$$
x \cdot y=e\left(e^{-1} x * e^{-1} y\right) .
$$


It follows that

$$
x \cdot y=\rho(x, y)=\left(\rho_{1}(x, y), \ldots, \rho_{k}(x, y)\right),
$$

where the $\rho_{j}^{\prime}$ s are rational polynomials of the form

$$
\begin{aligned}
\rho_{j}(x, y) & =x_{j}+y_{j}+\tau_{j}\left(x_{1}, \ldots, x_{j-1}, y_{1}, \ldots, y_{j-1}\right) \\
& =x_{j}+y_{j}+\sigma_{j}\left(x_{1}, \ldots, x_{j-1}, y_{1}, \cdots, y_{j-1}\right)+\cdots,
\end{aligned}
$$

and where $\sigma_{j}=\sigma_{j}\left(x_{1}, \ldots, y_{j-1}\right)$ denotes the quadratic part of $\tau_{j}$. Then $\mathbb{Q}^{k}$ can be given a Lie algebra structure with bracket [, ] defined by setting

$$
[x, y]=\sigma(x, y)-\sigma(y, x), \quad \text { where } \sigma(x, y)=\left(\sigma_{1}, \ldots, \sigma_{k}\right) .
$$

This bracket on $\mathbb{Q}^{k}$ induces the original Lie algebra structure on $\mathcal{L}\left(=\mathcal{G}_{\mathbb{Q}}\right)$ via the maps $e, \varphi$, and $\psi$.

\section{Finitely-generated torsion-free nilpotent groups.}

Let $G$ be a torsion-free, nilpotent group on $k$ generators. A set of elements $\left\{g_{1}, \ldots, g_{k}\right\}$ of $G$ is called a canonical basis for $G$ if every element of $G$ can be expressed in the form

$$
g_{1}^{x_{1}} \cdots g_{k}^{x_{k}}, \quad x_{j} \in \mathbb{Z}
$$

so that the elements of the form $g_{i}^{x_{i}} \cdots g_{k}^{x_{k}}$ generate a normal subgroup $G^{i}$ of $G$, for $i=1,2, \ldots, k$, with the quotients $G^{i} / G^{i+1}$ being infinite cyclic.

Any such group is isomorphic to a uniform discrete subgroup of the real Lie group $\mathcal{G}_{\mathbb{R}}$. This isomorphism is given as the composition of maps

$$
G \stackrel{i}{\longrightarrow} \mathcal{G} \stackrel{\varphi}{\longrightarrow} \mathcal{G}_{\mathbb{Q}} \stackrel{\text { inclusion }}{\longrightarrow} \mathcal{G}_{\mathbb{R}}
$$

where

$$
i\left(g_{1}^{x_{1}} \cdots g_{k}^{x_{k}}\right)=x_{1} g_{1} * \cdots * x_{k} g_{k}
$$

and

$$
\varphi\left(x_{1} g_{1} * \cdots * x_{k} g_{k}\right)=\xi_{1} g_{1}+\cdots+\xi_{k} g_{k} .
$$

As in Proposition 1, it follows that $\left\{g_{1}, \ldots, g_{k}\right\}$ is a canonical basis for the Lie algebra $\mathcal{L}$. Note that while the $x_{j}^{\prime}$ s are integers the $\xi_{j}^{\prime}$ s are rational numbers in general. Furthermore, recall that $\mathcal{G}_{\mathbb{Q}}=\mathcal{L}_{\mathbb{Q}}=\mathcal{L}$ and $\mathcal{G}_{\mathbb{R}}=\mathcal{L}_{\mathbb{R}}$ as sets. We identify $G$ with its image $i(G)$ in $\mathcal{G}$. Thus we have:

(i) $G$ is the subgroup of $\mathcal{G}$ consisting of the elements

$$
\left(x_{1} g_{1}\right) * \cdots *\left(x_{k} g_{k}\right), \quad x_{j} \in \mathbb{Z} .
$$

(ii) The groups $\mathcal{G}_{i}=G \cap \mathcal{G}^{i}$ of the elements

$$
\left(x_{i} g_{i}\right) * \cdots *\left(x_{k} g_{k}\right), \quad x_{j} \in \mathbb{Z}
$$

are normal subgroups of $G$. 
(iii) The quotient groups $\mathcal{G}_{i} / \mathcal{G}_{i+1}$ are infinite cyclic.

(iv) The $\mathbb{Z}$ module $\bigoplus_{i \geq 1} \mathcal{G}_{i} / \mathcal{G}_{i+1}$ has a graded Lie algebra structure induced by the commutators on $G$. This Lie algebra structure is induced from that on $\bigoplus_{i \geq 1} \mathcal{L}^{i} / \mathcal{L}^{i+1}$ by the map $\varphi \circ i: G \longrightarrow \mathcal{L}$.

(v) The group $G$, as a set, can be identified with $\mathbb{Z}^{k} \subset \mathbb{Q}^{k}$ via the map $i \circ e$. The group structure on $\mathbb{Z}^{k}$ in (3) is induced by $e$. Therefore, the rational polynomials $\rho_{j}$ are $\mathbb{Z}$-valued polynomials when restricted to $\mathbb{Z}^{k} \times \mathbb{Z}^{k}$. Each polynomial $\rho_{j}(x, y)$ can be written as a sum (over $\mathbb{Z}$ ) of binomials

$$
\left(\begin{array}{l}
x_{1} \\
a_{1}
\end{array}\right)\left(\begin{array}{l}
x_{2} \\
a_{2}
\end{array}\right) \cdots\left(\begin{array}{l}
x_{r} \\
a_{r}
\end{array}\right)\left(\begin{array}{l}
y_{1} \\
b_{1}
\end{array}\right)\left(\begin{array}{l}
y_{2} \\
b_{2}
\end{array}\right) \cdots\left(\begin{array}{l}
y_{s} \\
b_{s}
\end{array}\right),
$$

with $a_{u}, b_{v}$ nonnegative integers, $r, s \leq j$, where not all $a_{u}^{\prime}$ s and not all $b_{v}^{\prime}$ s are equal to zero and also as sum (over $\mathbb{Q}$ ) of polynomials (see $[1])$.

Thus, we can consider the group $\mathbb{Z}^{k}$ with the group operation given by (3). We denote by $\mathbb{G}$ the image of $\mathbb{Z}^{k}$ in $\mathcal{G}_{\mathbb{Q}}$ under the one-to-one map

$$
c h=\varphi \circ e^{-1}: \mathbb{Z}^{k} \longrightarrow \mathbb{G} \subset \mathcal{G}_{\mathbb{Q}} .
$$

Recall that, given a canonical basis $\left\{g_{1}, \ldots, g_{k}\right\}$ for $G, \mathcal{G}^{k}$ is the set of elements $\xi_{1} g_{1}+\cdots+\xi_{k} g_{k}$, where $\xi_{1}, \ldots, \xi_{k}$ are certain rational numbers. $\mathcal{G}^{k}$ with the operation $*$ given by the Campbell-Hausdorff formula is a group. It is important to note that the sequence of rational numbers $\xi_{1}, \ldots, \xi_{k}$ is not an arbitrary sequence of rational numbers; it depends on the choice of a canonical basis $\left\{g_{1}, \ldots, g_{k}\right\}$ for $G$. Once a canonical basis is chosen then $\xi_{j}=x_{j}+\varphi_{j}\left(x_{1}, \ldots, x_{j-1}\right)$, where the $x_{u}$ 's are integers and the rational polynomials $\varphi_{j}$ are determined by the Lie algebra bracket.

We summarize our observations as follows:

Proposition 2. Let $G$ be a torsion-free nilpotent group on $k$ generators, and let $\left\{g_{1}, \ldots, g_{k}\right\}$ be a canonical basis for $G$. Then the group $G$ is isomorphic to the group $\mathbb{Z}^{k}$ with the group operation defined by

$$
x \cdot y=\operatorname{ch}^{-1}(\operatorname{ch}(x) * \operatorname{ch}(y)),
$$

where $x=\left(x_{1}, \ldots, x_{k}\right), y=\left(y_{1}, \ldots, y_{k}\right)$ and where $*$ is given by the CampbellHausdorff formula (1).

In order to illustrate the type of rational numbers $\xi_{1}, \ldots, \xi_{k}$ that can occur, we look again at a classical example.

Example 1 (The Heisenberg group). Let $G$ be the group of matrices

$$
\left(\begin{array}{ccc}
1 & a_{1} & a_{3} / k \\
0 & 1 & a_{2} \\
0 & 0 & 1
\end{array}\right), \quad \text { where } a_{j} \in \mathbb{Z} \text { and } k \text { is a fixed integer }>0 .
$$


Then

$$
\left\{g_{1}=\left(\begin{array}{lll}
1 & 1 & 0 \\
0 & 1 & 0 \\
0 & 0 & 1
\end{array}\right), g_{2}=\left(\begin{array}{ccc}
1 & 0 & 0 \\
0 & 1 & 1 \\
0 & 0 & 1
\end{array}\right), g_{3}=\left(\begin{array}{ccc}
1 & 0 & 1 / k \\
0 & 1 & 0 \\
0 & 0 & 1
\end{array}\right)\right\}
$$

is a canonical basis, and

$$
g_{1}^{x_{1}} g_{2}^{x_{2}} g_{3}^{x_{3}}=\left(\begin{array}{ccc}
1 & x_{1} & \left(x_{3} / k\right)+x_{1} x_{2} \\
0 & 1 & x_{2} \\
0 & 0 & 1
\end{array}\right) .
$$

$\left\{g_{1}, g_{2}, g_{3}\right\}$ is a canonical basis for $\mathcal{L},\left[g_{1}, g_{2}\right]=k g_{3}$, and $\left[g_{i}, g_{j}\right]=0$ otherwise. We have

$$
\operatorname{ch}(x)=x_{1} g_{1}+x_{2} g_{2}+\left(x_{3}+\frac{k}{2} x_{1} x_{2}\right) g_{3}, \quad x=\left(x_{1}, x_{2}, x_{3}\right) ;
$$

therefore,

$$
\xi_{1}=x_{1}, \quad \xi_{2}=x_{2}, \quad \xi_{3}=x_{3}+\frac{k}{2} x_{1} x_{2} .
$$

It can be checked that the group structure on $\mathbb{Z}^{3}$ is given by (4). Namely,

$$
\begin{aligned}
\operatorname{ch}(x) * \operatorname{ch}(y)= & \left(x_{1}+y_{1}\right) g_{1}+\left(x_{2}+y_{2}\right) g_{2} \\
& +\left(\left(x_{3}+y_{3}-k y_{1} x_{2}\right)+\frac{k}{2}\left(x_{1}+y_{1}\right)\left(x_{2}+y_{2}\right)\right) g_{3} \\
= & \varphi\left(\left(\left(x_{1}+x_{2}\right) g_{1}\right) *\left(\left(y_{1}+y_{2}\right) g_{2}\right) *\left(\left(x_{3}+y_{3}-k y_{1} x_{2}\right) g_{3}\right)\right) \\
= & \varphi \circ e^{-1}(x \cdot y) \\
= & c h(x \cdot y) .
\end{aligned}
$$

As was mentioned above, a canonical basis for $G$ determines a canonical basis for the rational Lie algebra. However, the converse is not always true. A canonical basis for $\mathcal{L}$ is not always a canonical basis for $G$. This fact is demonstrated by the next example. We will see later that given a canonical basis for a Lie algebra, there is always a rationally equivalent basis that is induced by a canonical basis of the corresponding group.

Example 2. Let $\mathcal{L}$ be the Lie algebra over $\mathbb{Z}$ with generators $g_{1}, \ldots, g_{7}$ and with product given by

$$
\begin{aligned}
& {\left[g_{1}, g_{2}\right]=g_{4}-g_{5},} \\
& {\left[g_{1}, g_{3}\right]=g_{4}+g_{5}, \quad\left[g_{2}, g_{3}\right]=g_{5},} \\
& {\left[g_{1}, g_{4}\right]=g_{6}, \quad\left[g_{2}, g_{4}\right]=g_{6}, \quad\left[g_{3}, g_{4}\right]=g_{7} \text {, }} \\
& {\left[g_{1}, g_{5}\right]=g_{6}, \quad\left[g_{2}, g_{5}\right]=g_{7} \text {, }} \\
& {\left[g_{i}, g_{j}\right]=0 \quad \text { otherwise. }}
\end{aligned}
$$

$\mathcal{L}$ is a nilpotent finitely-generated Lie algebra with no torsion elements. The set $\left\{g_{1}, \ldots, g_{7}\right\}$ forms a canonical basis for $\mathcal{L}$ with $\left\{g_{i}, \ldots, g_{7}\right\}$ a basis for the ideal $\mathcal{L}^{i}$. We want to show that $\left\{g_{1}, \ldots, g_{7}\right\}$ can not be a canonical 
basis for a finitely-generated torsion-free nilpotent group whose associated Lie algebra is $\mathcal{L}$. The idea is the following. Assuming that $\left\{g_{1}, \ldots, g_{7}\right\}$ is such a canonical basis we show that there are elements $x, y \in \mathbb{Z}^{7}$ such that $\operatorname{ch}^{-1}(\operatorname{ch}(x) * \operatorname{ch}(y))$ fails to be a sequence of seven integers.

Let $x=\left(x_{1}, \ldots, x_{7}\right) \in \mathbb{Z}^{7}$. Then $\operatorname{ch}(x) \in \mathcal{G}_{\mathbb{Q}}$,

$$
\begin{aligned}
& \operatorname{ch}(x) \\
& =\varphi\left(x_{1} g_{1} * \cdots * x_{7} g_{7}\right) \\
& =x_{1} g_{1}+x_{2} g_{2}+x_{3} g_{3} \\
& \quad+\left(x_{4}+1 / 2\left(x_{1} x_{2}+x_{1} x_{3}\right)\right) g_{4}+\left(x_{5}+1 / 2\left(-x_{1} x_{2}+x_{1} x_{3}+x_{2} x_{3}\right)\right) g_{5} \\
& \quad+\left(x_{6}+1 / 12\left(-x_{1} x_{2}^{2}+2 x_{1}^{2} x_{3}+2 x_{1} x_{2} x_{3}+6 x_{1} x_{4}+6 x_{2} x_{4}+6 x_{2} x_{5}\right)\right) g_{6} \\
& \quad+\left(x_{7}+1 / 12\left(x_{1} x_{2}^{2}-x_{1} x_{3}^{3}+x_{2}^{2} x_{3}-2 x_{1} x_{2} x_{3}+6 x_{3} x_{4}+6 x_{2} x_{5}\right)\right) g_{7}
\end{aligned}
$$

and there is a similar formula when $x$ is replaced by $y=\left(y_{1}, \ldots, y_{7}\right)$.

Now we assume that there is a sequence of integers $z=\left(z_{1}, \ldots, z_{7}\right) \in \mathbb{Z}^{7}$ such that $\operatorname{ch}(z)=\operatorname{ch}(x) * \operatorname{ch}(y)$. A routine computation shows that

$$
\begin{aligned}
z_{i}= & x_{i}+y_{i}, \quad i=1,2,3 \\
z_{4}= & x_{4}+y_{4}-x_{2} y_{1}-x_{3} y_{1} \\
z_{5}= & x_{5}+y_{5}+x_{2} y_{1}-x_{3} y_{1}-x_{3} y_{2}, \\
z_{6}= & x_{6}+y_{6}-x_{4} y_{1}-x_{4} y_{2}-x_{5} y_{1} \\
& +x_{2} y_{1} y_{2}+x_{3} y_{1} y_{2}+x_{3} y_{1}^{2}+\frac{1}{2} x_{2}^{2} y_{1} \\
z_{7}= & x_{7}+y_{7}-x_{5} y_{2}-x_{4} y_{3}-x_{2} y_{1} y_{2}+x_{2} y_{1} y_{3}+x_{3} y_{1} y_{2} \\
& +x_{3} y_{1} y_{3}+x_{2} x_{3} y_{1}+\frac{1}{2} x_{3}^{2} y_{1}-\frac{1}{2} x_{2}^{2} y_{1} \frac{1}{2} x_{3} y_{2}^{2} .
\end{aligned}
$$

From here it is immediate that for $x$ and $y$ such that $x_{2}=1, x_{j}=0, j \neq 2$ and $y_{1}=1, y_{i}=0, i \neq 1$ we get $z_{6}=1 / 2$ and $z_{7}=-1 / 2$. Thus, the cannonical basis $\left\{g_{1}, \ldots, g_{7}\right\}$ for $\mathcal{L}$ is not induced by a cannonical basis for any group.

However, it is possible to find a nilpotent torsion-free Lie algebra $\mathcal{L}^{\prime}$ on seven generators $e_{1}, \ldots, e_{7}$ over $\mathbb{Z}$ such that $\mathcal{L}$ and $\mathcal{L}^{\prime}$ are isomorphic over $\mathbb{Q}$ (but not over $\mathbb{Z}$ ) and that the generators $e_{1}, \ldots, e_{7}$ are induced by a canonical basis for a torsion-free nilpotent group on 7 generators and that $\mathcal{L}^{\prime}$ is the corresponding Lie algebra. Such a Lie algebra is the following: 
Example 3. Let $\mathcal{L}^{\prime}$ be the Lie algebra over $\mathbb{Z}$ with generators $e_{1}, \ldots, e_{7}$ and products

$$
\begin{array}{rlrl}
{\left[e_{1}, e_{2}\right]} & =2 e_{4}-2 e_{5}, & & \\
{\left[e_{1}, e_{3}\right]} & =4 e_{4}+4 e_{5}, & & {\left[e_{2}, e_{3}\right]=2 e_{5},} \\
{\left[e_{1}, e_{4}\right]=2 e_{6},} & & {\left[e_{2}, e_{4}\right]=e_{6}, \quad\left[e_{3}, e_{4}\right]=2 e_{7},} \\
{\left[e_{1}, e_{5}\right]} & =2 e_{6}, & & {\left[e_{2}, e_{5}\right]=e_{7},} \\
{\left[e_{i}, e_{j}\right]=0} & & \text { otherwise. }
\end{array}
$$

In this case for $x=\left(x_{1}, \ldots, x_{7}\right)$ and $y=\left(y_{1}, \ldots, y_{7}\right)$ from $\mathbb{Z}^{7}, \operatorname{ch}(x)$ and $\operatorname{ch}(y)$ belong to $\mathbb{G}^{7} \subset \mathcal{G}_{\mathbb{Q}}$,

$$
\begin{aligned}
& \operatorname{ch}(x) \\
& =x_{1} e_{1}+x_{2} e_{2}+x_{3} e_{3} \\
& \quad+\left(x_{4}+2 x_{1} x_{3}+x_{1} x_{2}\right) e_{4}+\left(x_{5}+2 x_{1} x_{3}+x_{2} x_{3}-x_{1} x_{2}\right) e_{5} \\
& \quad+1 / 12\left(12 x_{6}+12 x_{1} x_{4}+6 x_{2} x_{4}+12 x_{1} x_{5}+8 x_{1} x_{2} x_{3}+16 x_{1}^{2} x_{3}-2 x_{1} x_{2}^{2}\right) e_{6} \\
& \quad+1 / 12\left(12 x_{7}+12 x_{3} x_{4}+6 x_{2} x_{5}-8 x_{1} x_{2} x_{3}-8 x_{1} x_{3}^{2}+2 x_{2}^{2} x_{3}+2 x_{1} x_{2}^{2}\right) e_{7} .
\end{aligned}
$$

There are similar formulas for $\operatorname{ch}(y)$. The group structure on $\mathbb{Z}^{7}$ is given by the formula $z=\operatorname{ch}^{-1}(\operatorname{ch}(x) * \operatorname{ch}(y))$,

$$
\begin{aligned}
z_{1}= & x_{1}+y_{1}, \\
z_{2}= & x_{2}+y_{2}, \\
z_{3}= & x_{3}+y_{3}, \\
z_{4}= & x_{4}+y_{4}-2 x_{2} y_{1}-4 x_{3} y_{1} \\
z_{5}= & x_{5}+y_{5}+2 x_{2} y_{1}-4 x_{3} y_{1}-2 x_{3} y_{1} \\
z_{6}= & x_{6}+y_{6}-2 x_{4} y_{1}-x_{4} y_{2}-2 x_{5} y_{1}+2 x_{2} y_{1} y_{2}+4 x_{3} y_{1} y_{2}+8 x_{3} y_{1}^{2}+x_{2}^{2} y_{1} \\
z_{7}= & x_{7}+y_{7}-x_{5} y_{2}-x_{4} y_{3}-2 x_{2} y_{1} y_{2}+4 x_{2} y_{1} y_{3}+4 x_{3} y_{1} y_{2}+8 x_{3} y_{1} y_{3} \\
& +4 x_{2} x_{3} y_{1}+4 x_{3}^{2} y_{1}-x_{2}^{2} y_{1}+x_{3} y_{2}^{2} .
\end{aligned}
$$

Thus, the group $G^{\prime}$ whose Lie algebra is $\mathcal{L}^{\prime}$ is the set of elements of the form $e_{1}^{x_{1}} * \cdots * e_{7}^{x_{7}}$ with the product given by the above formulas.

Given a finitely-generated, torsion-free, nilpotent Lie Algebra over the integers, there is a family of Lie algebras over the integers that are equivalent to it over the rationals. All such algebras can be provided with canonical bases. However, as we have seen in Example 2, not all such bases are determined by a canonical basis for a finitely-generated, torsion-free, nilpotent group. It seems natural to ask the following question: Suppose that we are given a finitely-generated nilpotent Lie algebra over the integers. Can we find a finitely-generated torsion-free nilpotent group and a canonical basis for such a group so that its associated Lie algebra is a Lie algebra over the integers that is rationally equivalent to the original one? It turns out that it 
is possible. In the last part of this paper we describe a procedure for finding such a group.

\section{The spectral sequence for polynomial cochains.}

In this part we start with a finitely-generated, torsion-free, nilpotent group $G$ with a canonical basis. The induced Lie algebra is a Lie algebra over the rationals together with the induced canonical basis. In [1] we proved that the integral cohomology of $G$ is isomorphic to the cohomology of the differential algebra $P(G)$ of rational polynomial maps from $\mathbb{Z}^{k}$ to $\mathbb{Z}$, where $k$ is the number of generators of the group $G$. The differential on $P(G)$ is induced by the group structure on $\mathbb{Z}$. In this section we use the structure of the group $G$ to define a filtration on $P(G)$, and study the resulting spectral sequence. Starting with the sequence of normal subgroups of $G, G=G^{1}>G^{2}>$ $\cdots>G^{k}>1$, denote by $i_{1}$ the largest positive integer such that $G^{1} / G^{i_{1}+1}$ is a torsion-free, abelian group and by $i_{2}$ the largest positive integer such that $G^{i_{1}+1} / G^{i_{2}+1}$ is a torsion-free, abelian group. Inductively, let $0=i_{0}<$ $i_{1}<i_{2}<\cdots<i_{N-1}<i_{N}=k$ be the largest positive integers such that $G^{i_{j-1}+1} / G^{i_{j}+1}, j=1,2, \ldots, N-1$, are torsion-free, abelian groups. Then

$$
G=G_{1}>G_{2}>\cdots>G_{N+1}=1, \quad G_{j+1}=G^{i_{j}+1}, \quad j=0,1,2, \ldots, N-1
$$

is the shortest central series with torsion-free quotients. Then $\varphi$ maps this sequence of normal subgroups to a sequence of ideals of the Lie algebra $\mathcal{L}$.

$$
\begin{gathered}
\mathcal{L}=\mathcal{L}_{1} \supset \mathcal{L}_{2} \supset \cdots \supset \mathcal{L}_{N+1}=0, \quad \mathcal{L}_{j+1}=\mathcal{L}^{i_{j}+1}, \\
\varphi\left(G_{j}\right) \subset \mathcal{L}_{j}, \quad j=1,2, \ldots, N .
\end{gathered}
$$

From (4) it follows that the commutators induce a Lie algebra structure on the $\mathbb{Z}$-module gr $G=\oplus_{j \geq 1} G_{j} / G_{j+1}$. The Lie bracket [, ] on $\mathcal{L}$ induces a Lie algebra structure on the $\mathbb{Q}$-module gr $\mathcal{L}=\oplus_{j \geq 1} \mathcal{L}_{j} / \mathcal{L}_{j+1}$. In addition the graded Lie algebras gr $G \otimes \mathbb{Q}$ and gr $\mathcal{L}$ are isomorphic. The isomorphism is induced by $\varphi$.

For the remainder of this paper, we assume that the canonical basis $\left\{g_{1}, \ldots, g_{k}\right\}$ for $G$ is such that $\left\{g_{1}, \ldots, g_{i_{1}}\right\}$ projects to a basis for $G_{1} / G_{2}$; $\left\{g_{i_{1}+1}, \ldots, g_{i_{2}}\right\}$ projects to a basis for $G_{2} / G_{3}$; and in general

$$
\left\{g_{i_{j-1}+1}, \ldots, g_{i_{j}}\right\} \quad \text { projects to a basis for } G_{j} / G_{j+1} \text {. }
$$

To each basis element $g_{j}$ of $g$ we assign a weight as follows. If $g_{j}$ projects to a basis element for $G_{r} / G_{r+1}$, then its weight is $\left|g_{j}\right|=r$. In particular, $\left|g_{i_{r-1}+1}\right|=\cdots=\left|g_{i_{r}}\right|=r$ for $r=1,2, \ldots$, and for every rational number $q$ we set

$$
\left|q g_{j}\right|=\left|g_{j}\right|
$$

Furthermore,

$$
\left|g_{r}+g_{s}\right|=\max \left\{\left|g_{r}\right|,\left|g_{s}\right|\right\}
$$


Since a canonical basis for $G$ induces a canonical basis for $\mathcal{L}$, we set the weight of an element $g_{j}$ of $\mathcal{L}$ to be the weight of $g_{j}$ considered as an element of $G$.

From (2) it follows that for any two elements of the canonical basis $g_{r} \in$ $\mathcal{L}_{a}, g_{s} \in \mathcal{L}_{b}$ the Lie bracket is

$$
\left[g_{r}, g_{s}\right]=\sum_{j \geq r+s} q_{j}^{r, s} g_{j},
$$

where $q_{j}^{r, s} \in \mathbb{Q}$ and $g_{j} \in \mathcal{L}_{a+b}$. Thus,

$$
\left|\left[g_{r}, g_{s}\right]\right| \geq\left|g_{r}\right|+\left|g_{s}\right| \text {. }
$$

The above summation goes over all $j$ such that

$$
\left|g_{j}\right| \geq\left|g_{r}\right|+\left|g_{s}\right| \text {. }
$$

Next we assign a weight to each binomial map $\mathbb{Z}^{k} \rightarrow \mathbb{Z}$ according to the rules:

$$
\begin{aligned}
\left|x_{j}\right| & =\left|g_{j}\right|, \\
\left|\left(\begin{array}{c}
x_{j} \\
a
\end{array}\right)\right| & =a\left|x_{j}\right|, \quad a=0,1,2, \ldots, \\
\left|p\left(\begin{array}{c}
x_{j} \\
a
\end{array}\right)\right| & =\left|\left(\begin{array}{c}
x_{j} \\
a
\end{array}\right)\right| \quad \text { for any integer } p \neq 0, \\
|u+v| & =\max \{|u|,|v|\}, \\
|u v| & =|u|+|v| .
\end{aligned}
$$

Analogously, we assign a weight to each monomial map $\mathbb{Z}^{k} \rightarrow \mathbb{Z}$ as follows:

$$
\begin{aligned}
\left|x_{j}\right| & =\left|g_{j}\right|, \\
\left|x_{j}^{a}\right| & =a\left|x_{j}\right|, \\
\left|q x_{j}\right| & =\left|x_{j}\right| \quad \text { for any rational number } q \neq 0, \\
|u+v| & =\max \{|u|,|v|\}, \\
|u v| & =|u|+|v| .
\end{aligned}
$$

Observe that each binomial of weight $w$ is a sum of monomials of weight $\leq w$ and that each monomial of weight $w$ is a sum of binomials of weight $\leq w$.

Lemma 1. Let the group structure on $G$ be induced by the --product (3). Then each $\rho_{j}(x, y)$ is a sum (over $\left.\mathbb{Z}\right)$ of binomials. $\rho_{j}(x, y)$ can be also expressed as a sum $($ over $\mathbb{Q})$ of monomials of weight $\leq\left|g_{j}\right|, j=1,2, \ldots$.

Proof. Because the group structure on $\mathbb{Z}^{k}$ is given by restricting the *product from $\mathcal{G}$ to $e^{-1}\left(\mathbb{Z}^{k}\right)$, it follows that the statement is proved if we verify that

$$
\left|\rho_{j}(x, y)\right| \leq\left|g_{j}\right|, \quad j=1,2, \ldots, k
$$


for every $x, y \in \mathbb{Z}^{k}$.

Equivalently, it suffices to show that the above inequality is satisfied in the product formula on $\mathcal{G}$,

$\left(\left(x_{1} g_{1}\right) * \cdots *\left(x_{k} g_{k}\right)\right) *\left(\left(y_{1} g_{1}\right) * \cdots *\left(y_{k} g_{k}\right)\right)=\left(\rho_{1}(x, y) g_{1} * \cdots * \rho_{k}(x, y) g_{k}\right)$.

But the maps $\varphi, \psi ; \mathcal{G} \underset{\psi}{\stackrel{\varphi}{\rightleftarrows}} \mathcal{L}$ are such that

$$
\begin{aligned}
& \psi\left(\xi_{1} g_{1}+\cdots+\xi_{k} g_{k}\right) \\
& =\left(\xi_{1} g_{1}\right) * \cdots *\left(\xi_{j}+\psi_{j}\left(\xi_{1}, \ldots, \xi_{j-1}\right)\right) g_{j} * \cdots *\left(\xi_{k}+\psi_{k}\left(\xi_{1}, \ldots, \xi_{k-1}\right)\right) g_{k} \\
& \varphi\left(x_{1} g_{1} * \cdots * x_{k} g_{k}\right) \\
& =x_{1} g_{1}+\cdots+\left(x_{j}+g_{j}\left(x_{1}, \ldots, x_{j-1}\right)\right) g_{j}+\cdots+\left(x_{k}+g_{k}\left(x_{1}, \ldots, x_{k-1}\right)\right) g_{k}
\end{aligned}
$$

where

$$
\left|\xi_{j}+\psi_{j}\left(\xi_{1}, \ldots, \xi_{j-1}\right)\right| \leq\left|\xi_{j}\right|=\left|g_{j}\right|
$$

and

$$
\left|x_{j}+\varphi_{j}\left(x_{1}, \ldots, x_{j-1}\right)\right| \leq\left|x_{j}\right|=\left|g_{j}\right| .
$$

It follows that the statement is proved if we show that the product in $\mathcal{L}$, given by (1)

$$
\begin{aligned}
\xi * \eta & =\left(\xi_{1} g_{1}+\cdots+\xi_{k} g_{k}\right) *\left(\eta_{1} g_{1}+\cdots+\eta_{k} g_{k}\right) \\
& =\left(\beta_{1}(\xi, \eta) g_{1}+\cdots+\beta_{k}(\xi, \eta) g_{k}\right)
\end{aligned}
$$

holds with $\beta_{j}(\xi, \eta)=\beta_{j}\left(\xi_{1}, \ldots, \xi_{j-1}, \eta_{1}, \ldots, \eta_{j-1}\right)$ and $\left|\beta_{j}(\xi, \eta)\right| \leq\left|g_{j}\right|$ for $j=1,2, \ldots, k$. But

$$
\beta_{j}(\xi, \eta)=\xi_{j}+\eta_{j}+\frac{1}{2}[\xi, \eta]_{j}+\cdots .
$$

Hence, it is enough to verify that $\left|[\xi, \eta]_{j}\right| \leq\left|g_{j}\right|$ if $\left|\xi_{j}\right| \leq\left|g_{j}\right|$ and $\left|\eta_{j}\right| \leq\left|g_{j}\right|$ for all $j=1,2, \ldots$.

Since

$$
\left[g_{r}, g_{s}\right]=\sum_{j \geq r+s} a_{j}^{r, s} g_{j}, \quad a_{j}^{r, s} \in \mathbb{Q},
$$

where the sum is over all $g_{j}^{\prime} s$ such that $\left|g_{j}\right| \geq\left|g_{r}\right|+\left|g_{s}\right|$ and

$$
[\xi, \eta]=\sum_{r, s=1}^{k} \sum_{j \geq r+s} \xi_{r} \eta_{s} a_{j}^{r, s} g_{j},
$$


the result follows from the identities

$$
\begin{aligned}
\left|[\xi, \eta]_{j}\right| & =\left|\sum_{\substack{1 \leq r, s \leq k \\
r+s \leq j}} \xi_{r} \eta_{s} a_{j}^{r, s}\right| \\
& \leq \max _{\substack{1 \leq r, s \leq k \\
r+s \leq j}}\left\{\left|\xi_{r} \eta_{s} a_{j}^{r, s}\right|\right\} \\
& =\max \left\{\left|\xi_{r} \eta_{s}\right|\right\} \\
& =\max \left\{\left|\xi_{r}\right|+\left|\eta_{s}\right|\right\} \\
& \leq \max \left\{\left|g_{r}\right|+\left|g_{s}\right|\right\} \leq\left|g_{j}\right| .
\end{aligned}
$$

Recall from [1] that an element of $P^{n}(G), n \geq 1$, is given as a linear combination, with integral coefficients, of the binomials

$$
\prod_{\substack{1 \leq i \leq k \\
1 \leq j \leq n}}\left(\begin{array}{c}
x_{i j} \\
a_{i j}
\end{array}\right),
$$

where the $a_{i j}^{\prime}$ s are nonnegative integers. $P^{0}(G)=\mathbb{Z}$. The differential $d$ on $P(G)$, as a derivation, is completely determined by its values on $P^{1}(G)$. On a typical binomial

$$
u=\left(\begin{array}{l}
x_{1} \\
a_{1}
\end{array}\right)\left(\begin{array}{l}
x_{2} \\
a_{2}
\end{array}\right) \cdots\left(\begin{array}{l}
x_{k} \\
a_{k}
\end{array}\right)
$$

in $P^{1}(G)$

$$
d u=\left(\begin{array}{c}
y_{1} \\
a_{1}
\end{array}\right) \cdots\left(\begin{array}{c}
y_{k} \\
a_{k}
\end{array}\right)+\left(\begin{array}{c}
z_{1} \\
a_{1}
\end{array}\right) \cdots\left(\begin{array}{c}
z_{k} \\
a_{k}
\end{array}\right)-\left(\begin{array}{c}
\rho_{1} \\
a_{1}
\end{array}\right) \cdots\left(\begin{array}{c}
\rho_{k} \\
a_{k}
\end{array}\right),
$$

where $\rho_{j}=y_{j}+z_{j}+\tau_{j}$, or more explicitly

$$
\rho_{j}\left(y_{1}, \ldots, y_{j}, z_{1}, \ldots, z_{j}\right)=y_{j}+z_{j}+\tau_{j}\left(y_{1}, \ldots, y_{j-1}, z_{1}, \ldots, z_{j-1}\right),
$$

where $\tau_{j}$ is a polynomial of degree $\geq 2$. To each polynomial cochain $u$ in $P(G)$ we assign a nonnegative integer $\|u\|$ called its norm, according to the following rules:

$$
\begin{aligned}
\left\|q g_{i}\right\| & =\left\|g_{i}\right\| \quad \text { for any rational number } q, \\
\left\|\left(\begin{array}{c}
x_{i} \\
a
\end{array}\right)\right\| & =a\left\|x_{i}\right\| \quad \text { for } i=1,2, \ldots, k, \\
\left\|p\left(\begin{array}{c}
x_{i} \\
a
\end{array}\right)\right\| & =\left\|\left(\begin{array}{c}
x_{i} \\
a
\end{array}\right)\right\| \quad \text { for any integer } p, \\
\|u v\| & =\|u\|+\|v\|, \\
\|u+v\| & =\max \{\|u\|,\|v\|\} \quad \text { for any } u \in P^{i}(G), v \in P^{j}(G) .
\end{aligned}
$$


Lemma 2. The polynomial functions $\rho_{j}$ and $\tau_{j}$ have their norms bounded from above. Namely,

$$
\begin{aligned}
\left\|\rho_{j}\left(x_{1}, \ldots, x_{j}, y_{1}, \ldots, y_{j}\right)\right\| & \leq\left|g_{j}\right|-1, \\
\left\|\tau_{j}\left(x_{1}, \ldots, x_{j-1}, y_{1}, \ldots, y_{j-1}\right)\right\| & \leq\left|g_{j}\right|-2 .
\end{aligned}
$$

Proof. $\rho_{j}\left(x_{1}, \ldots, x_{j}, y_{1}, \ldots, y_{j}\right)=x_{j}+y_{j}+\tau_{j}\left(x_{1}, \ldots, x_{j-1}, y_{1}, \ldots, y_{j-1}\right)$, where $\tau_{j}$ is a sum of binomials

$$
\beta=\left(\begin{array}{c}
x_{i_{1}} \\
a_{i_{1}}
\end{array}\right) \cdots\left(\begin{array}{l}
x_{i_{r}} \\
a_{i_{r}}
\end{array}\right)\left(\begin{array}{l}
y_{j_{1}} \\
b_{j_{1}}
\end{array}\right) \cdots\left(\begin{array}{c}
y_{j_{s}} \\
b_{j_{s}}
\end{array}\right),
$$

$r \geq 1, s \geq 1$, with integer coefficients. The norm of such a binomial $\beta$ is

$$
\begin{aligned}
\|\beta\| & =\sum a_{u}\left\|x_{u}\right\|+\sum b_{v}\left\|y_{v}\right\| \\
& =\sum a_{u}\left(\left|x_{u}\right|-1\right)+\sum b_{v}\left(\left|y_{v}\right|-1\right) \\
& \leq\left(\sum a_{u}\left|x_{u}\right|+\sum b_{v}\left|y_{v}\right|\right)-2 \\
& =|\beta|-2 \\
& \leq\left|\rho_{j}\right|-2 \\
& \leq\left|g_{j}\right|-2
\end{aligned}
$$

according to Lemma 1. Therefore, $\left\|\tau_{j}\right\| \leq\left|g_{j}\right|-2$. Since $\left\|\rho_{j}\right\|=$ $\max \left\{\left\|x_{j}\right\|,\|\beta\|\right\}$ over all binomials $\beta$ in $\rho_{j}$, it follows that $\left\|\rho_{j}\right\|=\left\|x_{j}\right\|$. Hence, $\left\|\rho_{j}\right\|=\left|g_{j}\right|-1$.

Using the norm \|\| , we define a filtration on $P(G)$ by setting

$$
\begin{aligned}
F^{i}= & F^{i} P(G)=\{u \in P(G) \mid\|u\| \leq i\}, \quad i=0,1,2, \ldots, \\
& F^{0} \subset F^{1} \subset F^{2} \subset \cdots F^{i-1} \subset F^{i} \subset \cdots \subset P(G) .
\end{aligned}
$$

This filtration has the following properties:

Lemma 3. (i) Under the cup product on chains

$$
F^{i} \times F^{j} \longrightarrow F^{i+j} \text {. }
$$

(ii) The differential d on $P(G)$ preserves the filtration; i.e.,

$$
d F^{i} \subset F^{i} .
$$

Proof. (i) follows immediately from the definition.

(ii) follows once we verify that for every binomial $u \in F^{i}, d u \in F^{i}$. Let

$$
u=\left(\begin{array}{l}
x_{1} \\
a_{1}
\end{array}\right) \cdots\left(\begin{array}{l}
x_{k} \\
a_{k}
\end{array}\right) .
$$


Then $d u$ is given by formula (5) above. Note that

$$
\left\|\left(\begin{array}{c}
y_{1} \\
a_{1}
\end{array}\right) \cdots\left(\begin{array}{c}
y_{k} \\
a_{k}
\end{array}\right)\right\|=\sum_{j=1}^{k} a_{j}\left(\left|g_{j}\right|-1\right) \leq i
$$

and

$$
\left\|\left(\begin{array}{c}
z_{1} \\
a_{1}
\end{array}\right) \cdots\left(\begin{array}{c}
z_{k} \\
a_{k}
\end{array}\right)\right\|=\sum_{j=1}^{k} a_{j}\left(\left|g_{j}\right|-1\right) \leq i .
$$

And according to Lemma 2,

$$
\left\|\left(\begin{array}{l}
\rho_{1} \\
a_{1}
\end{array}\right) \cdots\left(\begin{array}{c}
\rho_{k} \\
a_{k}
\end{array}\right)\right\|=\sum_{j=1}^{k} a_{j}\left\|\rho_{j}\right\| \leq \sum_{j=1}^{k} a_{j}\left(\left|g_{j}\right|-1\right),
$$

which is $\leq i$, by the assumption. Therefore, $\|d u\| \leq i$. Hence, $d u \in F^{i}$.

3.1. $E_{0}$ and $E_{1}$-terms of the spectral sequence. Let $\left\{E_{r}, d_{r}\right\}$ be the spectral sequence corresponding to the filtration $\left\{F^{i}\right\}$. In particular, we have

$$
E_{0}=F^{i} / F^{i-1}
$$

Let $p$ be the projection $F^{i} \rightarrow E_{0}$. The next step is to compute $E_{1}$. Since $d_{0}=p d$ and because each element of $F^{i}$ is an integral linear combination of binomials, we look more closely at the last term in formula (5) for $d u$, where $u \in F^{i}$.

$$
\begin{gathered}
\left(\begin{array}{c}
\rho_{1} \\
a_{1}
\end{array}\right) \cdots\left(\begin{array}{c}
\rho_{k} \\
a_{k}
\end{array}\right)=\left(\begin{array}{c}
y_{1}+z_{1}+\tau_{1} \\
a_{1}
\end{array}\right) \cdots\left(\begin{array}{c}
y_{k}+z_{k}+\tau_{k} \\
a_{k}
\end{array}\right) \\
\prod_{j=1}^{k}\left\{\left(\begin{array}{c}
y_{j}+z_{j} \\
a_{j}
\end{array}\right)+\left(\begin{array}{c}
\tau_{j} \\
a_{j}
\end{array}\right)+\sum_{r=1}^{a_{j}-1}\left(\begin{array}{c}
y_{j}+z_{j} \\
r
\end{array}\right)\left(\begin{array}{c}
\tau_{j} \\
a_{j}-r
\end{array}\right)\right\} .
\end{gathered}
$$

Observe that

$$
\left\|\tau_{j}\right\| \leq\left|g_{j}\right|-2
$$

by Lemma 2. Therefore,

$$
\left\|\left(\begin{array}{c}
\tau_{j} \\
a_{j}
\end{array}\right)\right\|=a_{j}\left\|\tau_{j}\right\|<a_{j}\left(\left|g_{j}\right|-1\right)
$$


and

$$
\begin{aligned}
& \left\|\sum_{r=1}^{a_{j}-1}\left(\begin{array}{c}
y_{j}+z_{j} \\
r
\end{array}\right)\left(\begin{array}{c}
\tau_{j} \\
a_{j}-r
\end{array}\right)\right\| \\
& =\max _{1 \leq r \leq a_{j}-1}\left\|\left(\begin{array}{c}
y_{j}+z_{j} \\
r
\end{array}\right)\left(\begin{array}{c}
\tau_{j} \\
a_{j}-r
\end{array}\right)\right\| \\
& =\max _{1 \leq r \leq a_{j}-1}\left\{r\left\|y_{j}+z_{j}\right\|+\left(a_{j}-r\right)\left\|\tau_{j}\right\|\right\} \\
& =\max _{1 \leq r \leq a_{j}-1}\left\{r\left\|y_{j}\right\|+\left(a_{j}-r\right)\left\|\tau_{j}\right\|, r\left\|z_{j}\right\|+\left(a_{j}-r\right)\left\|\tau_{j}\right\|\right\} \\
& =\max _{1 \leq r \leq a_{j}-1}\left\{r\left\|y_{j}\right\|+\left(a_{j}-r\right)\left\|\tau_{j}\right\|\right\}
\end{aligned}
$$

since $\left\|y_{j}\right\|=\left\|z_{j}\right\|$. Because $\left\|y_{j}\right\|=\left|g_{j}\right|-1$ and $\left\|\tau_{j}\right\|<\left|g_{j}\right|-1$, the last term is strictly smaller than

$$
\max _{1 \leq r \leq a_{j}-1}\left\{\left(r+\left(a_{j}-r\right)\right)\left(\left|g_{j}\right|-1\right)\right\}=a_{j}\left(\left|g_{j}\right|-1\right) .
$$

Therefore, the binomial $\left(\begin{array}{c}\rho_{1} \\ a_{1}\end{array}\right) \cdots\left(\begin{array}{c}\rho_{k} \\ a_{k}\end{array}\right)$ has the form

$$
\left(\begin{array}{c}
\rho_{1} \\
a_{1}
\end{array}\right) \cdots\left(\begin{array}{c}
\rho_{k} \\
a_{k}
\end{array}\right)=\prod_{j=1}^{k}\left\{X_{j}+T_{j}\right\}
$$

where

$$
\left\|X_{j}\right\|=a_{j}\left(\left|g_{j}\right|-1\right), \quad\left\|T_{j}\right\|<a_{j}\left(\left|g_{j}\right|-1\right) .
$$

But

$$
\prod_{j=1}^{k}\left(X_{j}+T_{j}\right)=\prod_{j=1}^{k} X_{j}+\sum T_{i_{1}} \cdots T_{i_{r}} X_{j_{1}} \cdots X_{j_{k-r}},
$$

where the sum is over all sequences $i_{1}, \ldots, i_{r}$ of elements from $1,2, \ldots, k$ of length $r \geq 1$ and where $j_{1}, \ldots, j_{k-r}$ is the complementary sequence. Observe that

$$
\begin{aligned}
& \left\|\sum T_{i_{1}} \cdots T_{i_{r}} X_{j_{1}} \cdots X_{j_{k-r}}\right\| \\
& =\max _{\text {(all sequences) }}\left\{\left\|T_{i_{1}} \cdots X_{j_{k-r}}\right\|\right\} \\
& <\max _{\text {(all sequences) }}\left\{a_{i_{1}}\left(\left|g_{i_{1}}\right|-1\right)+\cdots+a_{i_{r}}\left(\left|g_{i_{r}}\right|-1\right)\right. \\
& \left.\quad+a_{j_{1}}\left(\left|g_{j_{1}}\right|-1\right)+\cdots+a_{j_{k-r}}\left(\left|g_{j_{k-r}}\right|-1\right)\right\}
\end{aligned}
$$

which is strictly smaller than $i$ by the assumption that $u \in F^{i}$. Therefore,

$$
d_{0} u=p\left\{\prod_{j=1}^{k}\left(\begin{array}{c}
y_{j} \\
a_{j}
\end{array}\right)+\prod_{j=1}^{k}\left(\begin{array}{c}
z_{j} \\
a_{j}
\end{array}\right)-\prod_{j=1}^{k}\left(\begin{array}{c}
y_{j}+z_{j} \\
a_{j}
\end{array}\right)\right\}
$$


or $d_{0} u=p d_{1} u$, where $d_{1} u=0$ for $u=\left(\begin{array}{c}x_{i} \\ 1\end{array}\right)$ when $1 \leq i \leq k$ and

$$
d_{1} u=-\prod_{j=1}^{k} \prod_{r=1}^{a_{j}-1}\left(\begin{array}{c}
y_{j} \\
r
\end{array}\right)\left(\begin{array}{c}
z_{j} \\
a_{j}-r
\end{array}\right),
$$

when $a_{j} \geq 2$. But this is the differential on $P^{1}(A)$, when $A$ is the $\mathbb{Z}$-module $G_{1} / G_{2} \oplus \cdots \oplus G_{j} / G_{j+1} \oplus \cdots$ considered as an abelian group. Using the results of [1] , we obtain:

Theorem 1. The $E_{1}$-term of the spectral sequence $\left\{E_{r}, d_{r}\right\}$ is isomorphic to the exterior algebra $\bigwedge_{\mathbb{Z}}\left(\bar{x}_{1}, \ldots, \bar{x}_{k}\right)$ on one-dimensional generators.

3.2. $E_{2}$-term of the spectral sequence. Since the terms of the spectral sequence are algebras, it is sufficient to find the value of $d_{1}$ on the generators $\bar{x}_{j}$ of $\bigwedge_{\mathbb{Z}}\left(\bar{x}_{1}, \ldots, \bar{x}_{k}\right)$. If $x_{j},\left\|x_{j}\right\|=i$, is a representative of $\bar{x}_{j}$ then $d_{1} \bar{x}_{j}$ will be determined by the projection of $d x_{j}$ into the submodule of $P(G)$ of elements whose norm is $i-1$.

From the definition of $d$ on $P(G)$ it follows that

$$
d x_{j}=-\tau_{j}\left(y_{1}, \ldots, y_{j-1}, z_{1}, \ldots, z_{j-1}\right),
$$

where

$$
\begin{aligned}
& \tau_{j}\left(y_{1}, \ldots, y_{j-1}, z_{1}, \ldots, z_{j-1}\right) \\
& =\sum c\left(a_{1}, \ldots, a_{j-1}, b_{1}, \ldots, b_{j-1}\right)\left(\begin{array}{c}
y_{1} \\
a_{1}
\end{array}\right) \cdots\left(\begin{array}{c}
y_{j-1} \\
a_{j-1}
\end{array}\right)\left(\begin{array}{c}
z_{1} \\
b_{1}
\end{array}\right) \cdots\left(\begin{array}{c}
z_{j-1} \\
b_{j-1}
\end{array}\right),
\end{aligned}
$$

where the coefficients $c(\cdots)$ are integers and at least two of the nonnegative integers $a_{1}, \ldots, b_{j-1}$ are nonzero. Note that

$$
\begin{aligned}
\left\|\left(\begin{array}{c}
y_{1} \\
a_{1}
\end{array}\right) \cdots\left(\begin{array}{c}
z_{j-1} \\
b_{j-1}
\end{array}\right)\right\| & =\sum_{t=1}^{j-1}\left(a_{t}\left\|y_{t}\right\|+b_{t}\left\|z_{t}\right\|\right) \\
& =\sum_{t=1}^{j-1}\left(a_{t}+b_{t}\right)\left(\left|g_{t}\right|-1\right) \\
& =\sum_{t=1}^{j-1}\left(a_{t}+b_{t}\right)\left|g_{t}\right|-\sum_{t=1}^{j-1}\left(a_{t}+b_{t}\right) .
\end{aligned}
$$

We are looking for the sequences of nonnegative integers $a_{1}, \ldots, a_{j-1}$, $b_{1}, \ldots, b_{j-1}$ such that

$$
\left\|\left(\begin{array}{l}
y_{1} \\
a_{1}
\end{array}\right) \cdots\left(\begin{array}{c}
y_{j-1} \\
a_{j-1}
\end{array}\right)\left(\begin{array}{l}
z_{1} \\
b_{1}
\end{array}\right) \cdots\left(\begin{array}{c}
z_{j-1} \\
b_{j-1}
\end{array}\right)\right\|=i-1=\left|g_{j}\right|-2 .
$$

From the above identity, it follows that such sequences satisfy

$$
\sum_{t=1}^{j-1}\left(a_{t}+b_{t}\right)\left|g_{t}\right|-\sum_{t=1}^{j-1}\left(a_{t}+b_{t}\right)=\left|g_{t}\right|-2 .
$$


But from Lemma 1, it follows that

$$
\left|\left(\begin{array}{c}
y_{1} \\
a_{1}
\end{array}\right) \cdots\left(\begin{array}{c}
z_{j-1} \\
b_{j-1}
\end{array}\right)\right|=\sum_{t=1}^{j-1}\left(a_{t}\left|y_{t}\right|+b_{t}\left|z_{t}\right|\right)=\sum_{t=1}^{j-1}\left(a_{t}+b_{t}\right)\left|g_{t}\right| \leq\left|g_{j}\right| .
$$

Therefore,

$$
\sum_{t-1}^{j-1}\left(a_{t}+b_{t}\right)\left|g_{t}\right|=\sum_{t=1}^{j-1}\left(a_{t}+b_{t}\right)+\left|g_{j}\right|-2 \leq\left|g_{j}\right| .
$$

Hence, we must have

$$
\sum_{t=1}^{j-1}\left(a_{t}+b_{t}\right) \leq 2
$$

But if $\sum_{t=1}^{j-1}\left(a_{t}+b_{t}\right) \leq 1$, then there cannot be two nonzero integers among $a_{1}, \ldots, b_{j-1}$. Hence,

$$
\sum_{t=1}^{j-1}\left(a_{t}+b_{t}\right)=2
$$

But then exactly one of the $a_{t}$ 's and exactly one of the $b_{t}$ 's must be equal to 1 and all the other integers must be equal to 0 . Therefore, $d_{1} \bar{x}_{j}$ is determined by the terms in $\tau_{j}$ of the form

$$
\sum_{r, s=1}^{j-1} c_{j}^{r, s} x_{r} y_{s}, \quad c_{j}^{r, s} \in \mathbb{Z}
$$

where

$$
\left\|x_{r} y_{s}\right\|=\left\|x_{r}\right\|+\left\|y_{s}\right\|=\left|g_{j}\right|-2 .
$$

Theorem 2. The $E_{2}$-term of the spectral sequence $\left\{E_{r}, d_{r}\right\}$ is isomorphic to the integral cohomology of the graded Lie algebra

$$
\operatorname{gr} G=\bigoplus_{j \geq 1} G_{j} / G_{j+1}
$$

\section{The spectral sequence for the Lie algebra cochains.}

Let $G$ be a finitely-generated, torsion-free, nilpotent group with canonical basis $\left\{g_{1}, \ldots, g_{k}\right\}$. We denote by $\mathbb{A}$ the smallest subring of the rationals containing the structure constants of the corresponding Lie algebra $\mathcal{L}$. Let $\left\{\omega_{1}, \ldots, \omega_{k}\right\}$ be the $\mathbb{A}$-dual of the canonical basis $\left\{g_{1}, \ldots, g_{k}\right\}$ of $\mathcal{L}$. The algebra of $\mathbb{A}$-valued cochains on $\mathcal{L}$ is isomorphic to the exterior algebra

$$
\bigwedge\left(\mathcal{L}^{*}\right)=\bigwedge_{\mathbb{A}}\left\{\omega_{1}, \ldots, \omega_{k}\right\}
$$


$\mathcal{L}^{*}=\operatorname{Hom}_{\mathbb{A}}(\mathcal{L}, \mathbb{A})$. Recall that there is a pairing

$$
\langle,\rangle: \bigwedge^{r}\left(\mathcal{L}^{*}\right) \otimes \bigwedge^{r}(\mathcal{L}) \longrightarrow \mathbb{A}
$$

such that for every $v^{*}=v_{1}^{*} \wedge \cdots \wedge v_{r}^{*} \in \bigwedge^{r}\left(\mathcal{L}^{*}\right)$ and any $v=v_{1} \wedge \cdots \wedge v_{r} \in$ $\bigwedge^{r}(\mathcal{L})$

$$
\left\langle v^{*}, v\right\rangle=\operatorname{det}\left(\left\langle v_{i}^{*}, v_{j}\right\rangle\right) .
$$

In particular, for every $f \in \bigwedge^{r}\left(\mathcal{L}^{*}\right)$ and $g \in \bigwedge^{s}\left(\mathcal{L}^{*}\right), f \wedge g \in \bigwedge^{r+s}\left(\mathcal{L}^{*}\right)$ and

$$
\begin{aligned}
& \left\langle f \wedge g, v_{1} \wedge \cdots \wedge v_{r+s}\right\rangle \\
& =\sum_{r, s \text { shuffle }}(\operatorname{sgn} \pi)\left\langle f, v_{\pi(1)} \wedge \cdots \wedge v_{\pi(r)}\right\rangle\left\langle g, v_{\pi(r+1)} \wedge \cdots \wedge v_{\pi(r+s)}\right\rangle .
\end{aligned}
$$

For every $\omega=a_{1} \omega_{1}+\cdots+a_{k} \omega_{k}, a_{j} \in \mathbb{A}$, the differential $d: \mathcal{L}^{*} \longrightarrow \bigwedge^{2} \mathcal{L}^{*}$ takes the form

$$
d \omega=a_{1} \omega_{1}+\cdots+a_{r} \omega_{k}
$$

and extends to the map $d: \bigwedge^{r}\left(\mathcal{L}^{*}\right) \longrightarrow \bigwedge^{r+1}\left(\mathcal{L}^{*}\right)$ as a derivation.

Let \|\| be the norm on $\bigwedge_{\mathbb{A}}\left(\omega_{1}, \ldots, \omega_{k}\right)$ defined by the formulas

$$
\begin{gathered}
\left\|\omega_{j}\right\|=\left|g_{j}\right|-1, \quad\left\|q \omega_{j}\right\|=\left\|\omega_{j}\right\| \quad \text { for } q \in \mathbb{A}, \\
\left\|u^{*} \wedge v^{*}\right\|=\left\|u^{*}\right\|+\left\|v^{*}\right\|, \\
\left\|u^{*}+v^{*}\right\|=\max \left\{\left\|u^{*}\right\|+\left\|v^{*}\right\|\right\} .
\end{gathered}
$$

Using this norm, we define an ascending filtration on $\bigwedge\left(\mathcal{L}^{*}\right)$ by setting

$$
\mathcal{F}^{i}=\mathcal{F}^{i} \bigwedge\left(\mathcal{L}^{*}\right)=\left\{v^{*} \in \bigwedge\left(\mathcal{L}^{*}\right) \mid\left\|v^{*}\right\| \leq i\right\}
$$

Then

$$
\mathcal{F}^{0} \subset \mathcal{F}^{1} \subset \cdots \subset \mathcal{F}^{i} \subset \mathcal{F}^{i+1} \subset \cdots \subset \bigwedge\left(\mathcal{L}^{*}\right) .
$$

Lemma 4. The differential $d$ on $\Lambda\left(\mathcal{L}^{*}\right)$ lowers the filtration.

Proof. Since $d$ is a derivation it suffices to verify that if $\left\|\omega_{j}\right\|=i$, then $\left\|d \omega_{j}\right\|<i$. But

$$
\begin{aligned}
\left\|d \omega_{j}\right\| & =\max \left\{\left\|\omega_{r}\right\|+\left\|\omega_{s}\right\|\right\} \\
& =\max \left\{\left\|g_{r}\right\|+\left\|g_{s}\right\|\right\},
\end{aligned}
$$

where the max is taken over all pairs $\left\|g_{r}\right\|+\left\|g_{s}\right\| \leq\left\|g_{j}\right\|-1<\left\|g_{j}\right\|=i$. Thus, $d \mathcal{F}^{i} \subset \mathcal{F}^{i-1}, i \geq 1$. 
4.1. $\mathcal{E}_{0}, \mathcal{E}_{1}$, and $\mathcal{E}_{2}$ terms of the spectral sequence for Lie algebra cochains. We denote by $\left\{\mathcal{E}_{r}, d_{r}\right\}$ the spectral sequence associated with the above filtration. In particular, let

$$
\mathcal{E}_{0}^{i}=\mathcal{F}^{i} / \mathcal{F}^{i-1},
$$

and let $p: \mathcal{F}^{i} \longrightarrow \mathcal{F}^{i} / \mathcal{F}^{i-1}$ be the projection. Set $\bar{\omega}_{j}^{*}=p \omega_{j}^{*}$. Then $d_{0}$ on the element $\bar{\omega}_{j}$ with $\left\|\omega_{j}\right\|=i$ is determined by those elements in $d \omega_{j}$ which have norm exactly equal to $i$. But $\left\|\omega_{j}\right\| \leq i-1$. Hence, $d_{0} \equiv 0$.

Lemma 5. $\mathcal{E}_{1}$ is isomorphic to the exterior algebra $\bigwedge_{\mathbb{A}}\left(\bar{\omega}_{1}, \ldots, \bar{\omega}_{k}\right)$ on generators of dimension one.

The differential $d$ on $\bigwedge\left(\mathcal{L}^{*}\right)$ is dual to the Lie bracket in the sense that

$$
\begin{aligned}
\left\langle d \omega_{j}, g_{r} \wedge g_{s}\right\rangle & =-\left\langle\omega_{j},\left[g_{r}, g_{s}\right]\right\rangle \\
& =-\sum q_{i}^{r, s}\left\langle\omega_{j}, g_{i}\right\rangle \\
& =-\sum_{u<v} q_{j}^{u, v}\left\langle\omega_{u} \wedge \omega_{v}, g_{r} \wedge g_{s}\right\rangle .
\end{aligned}
$$

Note that the first summation is always zero if $\left|g_{j}\right|<\left|g_{r}\right|+\left|g_{s}\right|$ because $\left|g_{r}\right|+\left|g_{s}\right| \leq\left|g_{i}\right|$ and $\left\langle\omega_{j}, g_{i}\right\rangle=0$ whenever $\left|g_{j}\right|<\left|g_{i}\right|$. Hence,

$$
d \omega_{j}=-\sum_{r<s} q_{j}^{r, s} \omega_{r} \wedge \omega_{s}
$$

with $\left|g_{r}\right|+\left|g_{s}\right| \leq\left|g_{j}\right|$ or $\left|\omega_{r}\right|+\left|\omega_{s}\right| \leq\left|\omega_{j}\right|$, when $\left|\omega_{i}\right|=\left\|\omega_{i}\right\|+1$.

The differential $d_{1}$ on $\bar{\omega}_{j} \in \bigwedge_{\mathbb{A}}\left(\bar{\omega}_{1}, \ldots, \bar{\omega}_{k}\right)$ is determined by the terms in $\omega_{j}$ of the form

$$
\sum_{r<s} q_{j}^{r, s} \omega_{r} \wedge \omega_{s}
$$

where $\left\|g_{r}\right\|+\left\|g_{s}\right\|=\left\|g_{j}\right\|-1$ or equivalently $\left|g_{r}\right|+\left|g_{s}\right|=\left|g_{j}\right|$. This implies:

Theorem 3. The $\mathcal{E}_{2}$-term of the spectral sequence $\left\{\mathcal{E}_{r}, d_{r}\right\}$ is isomorphic to the cohomology of the graded Lie algebra

$$
\operatorname{gr} \mathcal{L}=\bigoplus_{j \geq 1}\left(\mathcal{L}_{j} / \mathcal{L}_{j+1} \otimes \mathbb{A}\right)
$$

Suppose that the group $G$ is two-stage nilpotent, and let $G=G_{1}>G_{2}>$ 1 be the shortest central series with torsion free quotients. Let $\left\{g_{1}, \ldots, g_{k}\right\}$ be a canonical basis such that $\left\{g_{1}, \ldots, g_{i}\right\}$ projects to a basis for $G_{1} / G_{2}$ and $\left\{g_{i+1}, \ldots, g_{k}\right\}$ projects to a basis for $G_{2}$. Then $\left\{g_{1}, \ldots, g_{k}\right\}$ determines a canonical basis for the induced Lie algebra $\mathcal{L}$. We use the same symbols for the canonical bases of the group and its associated Lie algebra. Then there is a sequence of ideals of $\mathcal{L}, \mathcal{L}=\mathcal{L}_{1} \supset \mathcal{L}_{2} \supset 0$ and the subset $\left\{g_{1}, \ldots, g_{i}\right\}$ of the canonical basis for $\mathcal{L}$ projects to a basis for $\mathcal{L}_{1} / \mathcal{L}_{2}$ and the subset $\left\{g_{i+1}, \ldots, g_{k}\right\}$ projects to a basis for $\mathcal{L}_{2}$. 
Note that for the elements of the canonical basis for $G$ and $\mathcal{L}$, the norm is

$$
\left\|g_{a}\right\|=0 \quad \text { for } a=1, \ldots, i ; \quad\left\|g_{b}\right\|=1 \quad \text { for } b=i+1, \ldots, k,
$$

and there are no elements of norm higher than 1 . Note that the differential $d x_{j}$ on $x_{j}$ in $P^{1}(G)$ can be written in the form

$$
d x_{j}=\delta_{0}\left(x_{j}\right)+\delta_{1}\left(x_{j}\right)+\cdots+\delta_{r}\left(x_{j}\right)+\cdots,
$$

where $\delta_{r}\left(x_{j}\right)$ is the element of the submodule of $P(G)$ whose norm is $\left\|x_{j}\right\|-r$. The differential $d_{r}$ in the spectral sequence $E_{r}$ for the group $G$ is determined by $\delta_{r}\left(x_{j}\right)$. Thus, in the case of a two-stage nilpotent group $d_{r}=0$ for $r \geq 2$. A similar argument shows that the differentials $d_{r}$ for $r \geq 2$ for the Lie algebra spectral sequence $\mathcal{E}_{r}$ are also zero. Therefore, the spectral sequence $\left\{E_{r}, d_{r}\right\}$ converges to

$$
H^{*}(G ; \mathbb{Z}) ; \text { i.e., } E_{2} \cong E_{\infty} \cong \operatorname{gr} H^{*}(G ; \mathbb{Z}),
$$

where $\operatorname{gr} H^{*}(G ; \mathbb{Z})$ is the graded module associated with the filtration $\left\{F^{i}\right\}$ of $P(G)$. If the Lie algebra $\mathcal{L}$ is a Lie algebra over the integers, then $\mathbb{A}=\mathbb{Z}$ and we can also conclude that the spectral sequence $\left\{\mathcal{E}_{r}, d_{r}\right\}$ converges to $H^{*}(\mathcal{L} ; \mathbb{Z})$; i.e.,

$$
\mathcal{E}_{2} \cong \mathcal{E}_{\infty} \cong \operatorname{gr} H^{*}(\mathcal{L} ; \mathbb{Z})
$$

Therefore, from Theorem 2 and Theorem 3 we obtain:

Corollary 1. Let $G$ be a two-stage, nilpotent, finitely-generated, torsionfree group, and let $\mathcal{L}$ be its Lie algebra such that $\mathcal{L}$ is a Lie algebra over the integers. Then there is an isomorphism of graded modules

$$
\operatorname{gr} H^{*}(G ; \mathbb{Z}) \cong \operatorname{gr} H^{*}(\mathcal{L} ; \mathbb{Z}) .
$$

If we do not assume that $G$ is a two-stage nilpotent group and that the associated Lie algebra $\mathcal{L}$ is over the integers, then we get the following:

Corollary 2. Let $\mathbb{A}$ be the smallest subring of rationals containing the structure constants of the Lie algebra $\mathcal{L}$. Then there is an isomorphism of $\mathbb{A}$ modules

$$
E_{2} \otimes \mathbb{A} \cong \mathcal{E}_{2} \cong H^{*}(\operatorname{gr} \mathcal{L} ; \mathbb{A}) .
$$

The conclusion of Corollary 1 is valid even for certain (perhaps for all) three-stage, nilpotent, finitely-generated, torsion-free groups. However, the argument based only on the length of lower central series used in the proof of the Corollary 1 is not sufficiently strong to prove the statement. An explicit computation of the $E_{2}$-term and of the differential $d_{2}$ is needed even in the example below.

Example 3 (continued). Let $G^{\prime}$ and $\mathcal{L}^{\prime}$ be the group and its associated Lie algebra as stated above. Recall that $\left\{g_{1}, \ldots, g_{7}\right\}$ is a canonical basis for $G^{\prime}$ and also for $\mathcal{L}^{\prime}$ (as we keep the same notation for both). The shortest 
lower central series with torsion-free quotients is $G=G_{1}>G_{2}>G_{3}>1$. Then $\left\{g_{1}, g_{2}, g_{3}\right\}$ projects to a basis of $G_{1} / G_{2},\left\{g_{4}, g_{5}\right\}$ projects to a basis of $G_{2} / G_{3}$, and $\left\{g_{6}, g_{7}\right\}$ projects to a basis of $G_{3}$. The Lie algebra $\mathcal{L}^{\prime}$ is filtered by the ideals $\mathcal{L}^{\prime}=\mathcal{L}_{1}^{\prime} \supset \mathcal{L}_{2}^{\prime} \supset \mathcal{L}_{3}^{\prime} \supset 0$ with appropriate quotients. The weights are $\left\|g_{1}\right\|=\left\|g_{2}\right\|=\left\|g_{3}\right\|=0,\left\|g_{4}\right\|=\left\|g_{5}\right\|=1,\left\|g_{6}\right\|=\left\|g_{7}\right\|=2$.

Since the comparison of Theorems 2 and 3 implies that the $E_{2}$-term of the spectral sequence for the group $G^{\prime}$ and the $\mathcal{E}_{2}$-term of the spectral sequence for the Lie algebra $\mathcal{L}^{\prime}$ are isomorphic $\mathbb{Z}$-modules, it suffices to compute only one of them. We choose to compute the $\mathcal{E}_{2}$-term for $\mathcal{L}^{\prime}$.

Let $\left\{\omega_{1}, \ldots, \omega_{7}\right\}$ be the $\mathbb{Z}$-dual basis of the canonical basis $\left\{g_{1}, \ldots, g_{7}\right\}$ of $\mathcal{L}^{\prime}$. Then the exterior algebra

$$
\bigwedge=\bigwedge\left(\mathcal{L}^{\prime *}=\operatorname{Hom}_{\mathbb{Z}}\left(\mathcal{L}^{\prime}, \mathbb{Z}\right)\right)=\bigwedge\left(\omega_{1}, \ldots, \omega_{7}\right)
$$

is filtered and graded. The elements $\omega_{1}, \ldots, \omega_{7}$ have dimension one and filtration $\left\|\omega_{1}\right\|=\left\|\omega_{2}\right\|=\left\|\omega_{3}\right\|=0,\left\|\omega_{4}\right\|=\left\|\omega_{5}\right\|=1,\left\|\omega_{6}\right\|=\left\|\omega_{7}\right\|=2$. The increasing filtration on the $\mathbb{Z}$-module $\bigwedge^{q}$ of elements of $\bigwedge$ of dimension $q$ is defined as the sequence of modules

$$
\mathcal{F}^{p} \bigwedge^{q}=\left\{\omega \in \bigwedge^{q} \mid\|\omega\| \leq p\right\}
$$

Setting $\mathcal{F}_{-p} \bigwedge^{q}=\mathcal{F}^{p} \bigwedge^{q}$, we get a descending filtration

$$
\mathcal{F}_{0} \bigwedge^{q} \subset \mathcal{F}_{-1} \bigwedge^{q} \subset \cdots \subset \mathcal{F}_{-p+1} \bigwedge^{q} \subset \cdots \subset \bigwedge^{q}
$$

Then

$$
\mathcal{E}_{0}^{-p, q}=\mathcal{F}_{-p} \bigwedge^{q-p} / \mathcal{F}_{-p+1} \bigwedge^{q-p}
$$

with $d_{0}=0$. Thus, $\mathcal{E}_{1}^{-p, q} \cong \mathcal{E}_{0}^{-p, q}$ and $d_{1}$ is determined by the full differential $d$ on $\bigwedge$. From the explicit formulas below we can see that $\left\|d \omega_{j}\right\|=\left\|\omega_{j}\right\|-1$. Since the differential $d$ is dual to the Lie bracket, we get

$$
\begin{aligned}
& d \omega_{1}=d \omega_{2}=d \omega_{3}, \\
& d \omega_{4}=2 \omega_{2} \omega_{1}+4 \omega_{3} \omega_{1}, \\
& d \omega_{5}=-2 \omega_{2} \omega_{1}+4 \omega_{3} \omega_{1}+2 \omega_{3} \omega_{2}, \\
& d \omega_{6}=2 \omega_{4} \omega_{1}+2 \omega_{5} \omega_{1}+\omega_{4} \omega_{2}, \\
& d \omega_{7}=\omega_{5} \omega_{2}+2 \omega_{4} \omega_{3} .
\end{aligned}
$$


Then an explicit computation shows that

$$
\begin{array}{rlrl}
\mathcal{E}_{2}^{0, q}=0, & & 4 \leq q, & \\
\mathcal{E}_{2}^{-1, q}=0, & q=2, & & 5 \leq q, \\
\mathcal{E}_{2}^{-2, q}=0, & q=3, & 7 \leq q, \\
\mathcal{E}_{2}^{-3, q}=0, & q \leq 5, & 9 \leq q, \\
\mathcal{E}_{2}^{-4, q}=0, & q \leq 7, & 10 \leq q, \\
\mathcal{E}_{2}^{-5, q}=0, & q \leq 9, & 12 \leq q, \\
\mathcal{E}_{2}^{-6, q}=0, & q \leq 10 & \\
\mathcal{E}_{2}^{-p, q}=0, & & p \geq 7 . &
\end{array}
$$

$d_{2}$ is determined by that part of the differential $d$ that lowers the filtration by two. But in our case $d$ lowers the filtration only by one. Thus $d_{2}=0$, and $\mathcal{E}_{\infty} \cong \mathcal{E}_{2} \cong \operatorname{gr} H^{*}\left(\mathcal{L}^{\prime} ; \mathbb{Z}\right)$. But, that is not the case for the differential $d$ on $P\left(G^{\prime}\right)$. That differential does have a component that lowers the degree by two. However, since there is an isomorphism $E_{2} \cong \mathcal{E}_{2}$ of the second terms of the spectral sequences for the group $G^{\prime}$ and for the Lie algebra $\mathcal{L}^{\prime}, d_{2}=0$ for dimensional reasons in all cases except possibly for $d_{2}: E_{2}^{-2,4} \longrightarrow E_{2}^{0,3}$ because $E_{2}^{-2,4} \cong \mathcal{E}_{2}^{-2,4} \cong \mathbb{Z}[\alpha]$, where $\alpha$ is represented by the element $\omega_{5} \omega_{4}-$ $4 \omega_{6} \omega_{1}-3 \omega_{6} \omega_{2}-2 \omega_{6} \omega_{3}-4 \omega_{7} \omega_{1}-2 \omega_{7} \omega_{2}$, and $E_{2}^{0,3} \cong \mathcal{E}_{2}^{0,3} \cong \mathbb{Z}_{2}[\beta]$, where $\beta$ is represented by $\omega_{3} \omega_{2} \omega_{1}$. In order to compute the differential $d_{2}$ we need an explicit form of the isomorphism $E_{2} \cong \mathcal{E}_{2}$. This isomorphism is induced by the map sending $\omega_{j}$ to the polynomial $x_{j}$ in $P\left(G^{\prime}\right)$ and the products $\omega_{j_{1}} \omega_{j_{2}} \cdots \omega_{j_{s}}$ to the monomials $x_{j_{1}} x_{j_{2}} \cdots x_{j_{s}}$ where $j_{1}>j_{2}>\cdots>j_{s}$. The technique of [1] is then used to show that $d_{2}=0$. Thus, $E_{3} \cong E_{2}$ and $d_{3}=0$ for dimensional reasons. In fact all the higher differentials are also zero for dimensional reasons. Then we get $E_{\infty} \cong \operatorname{gr} H^{*}\left(G^{\prime} ; \mathbb{Z}\right)$ and the comparison of Theorems 2 and 3 gives $\operatorname{gr} H^{*}\left(G^{\prime} ; \mathbb{Z}\right) \cong \operatorname{gr} H^{*}\left(\mathcal{L}^{\prime} ; \mathbb{Z}\right)$.

\section{Construction of a group from a Lie algebra.}

In his section we describe a method for generating examples of groups starting with Lie algebras. More precisely, let $\mathcal{L}$ be a nilpotent torsion-free Lie algebra over $\mathbb{Z}$ on a finite set of generators $\left\{g_{1}, \ldots, g_{k}\right\}$. Our aim is to find a torsion-free nilpotent group $G$ on $k$ generators whose associated Lie algebra $\mathcal{L}(G)$ is a Lie algebra over the integers. Then a canonical basis for $G$ induces a canonical basis for $\mathcal{L}(G)$ and the group structure on $G$ is determined via the group structure on $\mathbb{Z}^{k}$ by the formula $x \cdot y=\operatorname{ch}^{-1}(\operatorname{ch}(x) * \operatorname{ch}(y))$. In some instances $\mathcal{L}(G)=\mathcal{L}$ (see Example 1 and Example 3 ). Example 2 shows that it is not always the case. We will show below that there is always a 
group $G$ such that $\mathcal{L}(G)$ is rationally equivalent to $\mathcal{L}$. However, $G$ is not uniquely determined by $\mathcal{L}$.

Starting with a Lie algebra $\mathcal{L}$ with a canonical basis $\left\{g_{1}, \ldots, g_{k}\right\}$, we first check to see whether the basis is induced by a canonical basis of some group $G$.

Assume that $G$ is the set of elements $g_{1}^{x_{1}} \ldots, g_{k}^{x_{k}}, x=\left(x_{1}, \ldots, x_{k}\right) \in \mathbb{Z}^{k}$. Then compute $\operatorname{ch}(x)$ and $\operatorname{ch}(y)$ for $x, y \in \mathbb{Z}^{k}$. Note that $\operatorname{ch}(x)$ is completely determined by the Lie algebra via the Campbell-Hausdorff formula (1), and hence, so is the product $\operatorname{ch}(x) * \operatorname{ch}(y)$. Since $\operatorname{ch}: \mathbb{Z}^{k} \longrightarrow \mathbb{G}^{k}$ is a one-to-one map, it is enough to check whether or not $\operatorname{ch}(x) * \operatorname{ch}(y)$ has the form $\operatorname{ch}(z)$ for some $z \in \mathbb{Z}^{k}$ and for any choice of $x$ and $y$. If the answer is yes, then $G$ is the desired group and $\left\{g_{1}, \ldots, g_{k}\right\}$ is a canonical basis for $G$ induced by the given basis $\left\{g_{1}, \ldots, g_{k}\right\}$ for $\mathcal{L}$. This was the case in Example 3.

However, if there are elements $x, y \in \mathbb{Z}^{k}$ such that $\operatorname{ch}(x) * \operatorname{ch}(y)$ is not equal to $\operatorname{ch}(z)$ for some $z \in \mathbb{Z}^{k}$, then we proceed as follows: Let $\xi=\xi_{1} g_{1}+\cdots+\xi_{k} g_{k}$ and $\eta=\eta_{1} g_{1}+\cdots+\eta_{k} g_{k}$ be any two elements of $\mathcal{L}_{\mathbb{Q}}=$ the Lie algebra $\mathcal{L}$ over $\mathbb{Z}$, considered as a Lie algebra over $\mathbb{Q}$; i.e., $\xi_{j}, \eta_{j}$ are rational numbers. Then by (1)

$$
\begin{aligned}
\xi * \eta & =\beta_{1}(\xi, \eta) g_{1}+\cdots+\beta_{k}(\xi, \eta) g_{k} ; \\
\beta_{j}(\xi, \eta) & =\beta_{j}\left(\xi_{1}, \ldots, \xi_{j-1}, \eta_{1}, \ldots, \eta_{j-1}\right) .
\end{aligned}
$$

Let $N_{j}$ be the smallest positive integer such that

$$
\beta_{j}(\xi, \eta)=\frac{1}{N_{j}} B_{j}(\xi, \eta) ; \quad B_{j}(\xi, \eta)=B_{j}\left(\xi_{1}, \ldots, \xi_{j-1}, \eta_{1}, \ldots, \eta_{j-1}\right),
$$

where $B_{j}(\xi, \eta)$ is a polynomial with integer coefficients. $\left\{g_{1}, \ldots, g_{k}\right\}$ is assumed to be a canonical basis for $\mathcal{L}$. Therefore, $\left\{g_{j}, \ldots, g_{k}\right\}$ generates an ideal of $\mathcal{L}$, where $\mathcal{L}=\mathcal{L}_{1} \supset \mathcal{L}_{2} \supset \cdots$ is the shortest sequence of such ideals with abelian quotients. Then $\left\{g_{1}, \ldots, g_{k}\right\}$ can be grouped into subsets such that $\left\{g_{i_{j-1}+1}, \ldots, g_{i_{j}}\right\}$ projects to a basis for $\mathcal{L}_{j} / \mathcal{L}_{j+1}, j=1,2, \ldots$ Then we define the norm of each such element $g_{s}, i_{j-1} \leq s \leq i_{j}$, to be $\left\|g_{s}\right\|=s-1$. We assume that the norm satisfies the identities: $\left\|p g_{j}\right\|=\left\|g_{j}\right\|$ for any integer $p, 1 \leq j \leq k$, and $\|u+v\|=\max \{\|u\|,\|v\|\}$ for any $u, v \in \mathcal{L}$.

Furthermore, we set $\left\|\xi_{j}\right\|=\left\|g_{j}\right\|, 1 \leq j \leq k$ and $\left\|\xi_{r} \xi_{s}\right\|=\left\|\xi_{r}\right\|+\left\|\xi_{s}\right\|$, for any polynomial variables. Therefore, $\left\|B_{j+1}(\xi, \eta)\right\|=\alpha_{i_{1}+1}\left\|\xi_{i_{1}+1}\right\|+\cdots+$ $\alpha_{j}\left\|\xi_{j}\right\|$ (note that $\left\|\xi_{1}\right\|=\cdots=\left\|\xi_{i_{1}}\right\|=0$ ). Denote the sum of the coefficients by

$$
\varepsilon_{j}=\alpha_{i_{1}+1}+\cdots+\alpha_{j} .
$$

Now we define the subset $D$ of the $\mathbb{Q}$-vector space $\mathcal{L}_{\mathbb{Q}}$ to be the set of elements

$$
\frac{m_{1}}{n_{1}} g_{1}+\frac{m_{2}}{n_{2}} g_{2}+\cdots+\frac{m_{k}}{n_{k}} g_{k},
$$


where $m_{1}, \ldots, m_{k}$ are arbitrary integers and

$$
n_{j+1}=n_{j}^{\varepsilon_{j}} N_{j+1}, \quad j=1,2, \ldots .
$$

$n_{1}$ can be any nonzero integer. We choose $n_{1}=1$. It follows that the operation $*$ induces on $D$ the structure of a group. $D$ is a uniform discrete subgroup of the real Lie group $\mathcal{G}_{\mathbb{R}}$ and the quotient is a nilmanifold. The above construction of $D$ is similar to that given by Malcev [3]. For different choices of a canonical basis for $D$ we get different but rationally equivalent Lie algebras.

Now we make a choice of a canonical basis for $D$ that the associated Lie algebra is a Lie algebra over the integers. Starting with the original canonical basis $\left\{g_{1}, \ldots, g_{k}\right\}$ for $\mathcal{L}$ we define

$$
e_{j}=\frac{1}{n_{j}} g_{j}, \quad j=1,2, \ldots, k .
$$

Note that for every $r$ and $s,\left[g_{r}, g_{s}\right]=\sum q_{u}^{r, s} g_{u}$, where the summation is over all $u$ such that $\left\|g_{r}\right\|+\left\|g_{s}\right\|<\left\|g_{u}\right\|$. Therefore, $u>\max \{r, s$,$\} . Hence,$

$$
\begin{aligned}
{\left[e_{r}, e_{s}\right] } & =\frac{1}{n_{r} n_{s}}\left[g_{r}, g_{s}\right]=\frac{1}{n_{r} n_{s}} \sum q_{u}^{r, s} g_{u} \\
& =\frac{n_{u}}{n_{r} n_{s}} \sum q_{u}^{r, s} \frac{g_{u}}{n_{u}}=\frac{n_{u}}{n_{r} n_{s}} \sum q_{u}^{r, s} e_{u},
\end{aligned}
$$

where the $q_{u}^{r, s}$ are integers and $\frac{n_{u}}{n_{r} n_{s}}$ is also an integer since $u>\max \{r, s\}$. Therefore, the new Lie algebgra $\widehat{\mathcal{L}}$ is a Lie algebra over the integers that is rationally equivalent to $\mathcal{L}$. Then the group $G$ is defined as the set of elements $\left\{e_{1}^{x_{1}}, \ldots, e_{k}^{x_{k}}\right\}$ with the operation $\left(e_{1}^{x_{1}} \cdots e_{k}^{x_{k}}\right) \cdot\left(e_{1}^{y_{1}} \cdots e_{k}^{y_{k}}\right)=e_{1}^{z_{1}} \cdots e_{k}^{z_{k}}$ given by the formula $z=\operatorname{ch}^{-1}(\operatorname{ch}(x) * \operatorname{ch}(y))$.

Example 2 (continued). The new Lie algebra $\widehat{\mathcal{L}}$, in this case, has canonical basis $\left\{e_{1}=g_{1}, e_{2}=g_{2}, e_{3}=g_{3}, e_{4}=\frac{1}{2} g_{4}, e_{5}=\frac{1}{2} g_{5}, e_{6}=\frac{1}{12} g_{6}, e_{7}=\frac{1}{12} g_{7}\right\}$ and bracket

$$
\begin{aligned}
{\left[e_{1}, e_{2}\right] } & =2 e_{4}-2 e_{5} \\
{\left[e_{1}, e_{3}\right] } & =2 e_{4}+2 e_{5} \\
{\left[e_{1}, e_{4}\right] } & =6 e_{6} \\
{\left[e_{1}, e_{5}\right] } & =6 e_{6} \\
{\left[e_{i}, e_{j}\right] } & =0
\end{aligned}
$$

$$
\begin{aligned}
& {\left[e_{2}, e_{3}\right]=2 e_{5}} \\
& {\left[e_{2}, e_{4}\right]=6 e_{6} \quad\left[e_{3}, e_{4}\right]=6 e_{7}} \\
& {\left[e_{2}, e_{5}\right]=6 e_{7}} \\
& \text { otherwise. }
\end{aligned}
$$

The group $G$ is the set of elements $\left\{e_{1}^{x_{1}}, \ldots, e_{7}^{x_{7}}\right\}$ with the product induced by the formula $\operatorname{ch}^{-1}(\operatorname{ch}(x) * \operatorname{ch}(y))$. 


\section{References}

[1] B. Cenkl and R. Porter, Polynomial cochains on nilmanifolds, preprint.

[2] P. Hall, Nilpotent groups, Queens Mary College, Mathematics Notes, 1969.

[3] A. Malcev, On a class of homogeneous spaces, Izv. Akad. Nauk SSSR Ser. Mat., 13 (1949), 9-32 (Russian); English transl. in Math. USSR-Izv., 39 (1949).

Received June 11, 1998.

Department of Mathematics

NORTHEASTERN UNIVERSITY

Boston, MA 02115-5096

E-mail address: cenkl@neu.edu

Department of Mathematics

NORTHEASTERN UNIVERSITY

Boston, MA 02115-5096

E-mail address: rdp@neu.edu 